\title{
ZUM TEXT
}

Der vorliegende Text wurde aus Martin L. Wests Ilias-Edition in der Bibliotheca scriptorum Graecorum et Romanorum Teubneriana (Stuttgart/Leipzig/München 1998/2000) übernommen. Für die Belange des Kommentars hat Martin West die wichtigsten Hinweise zur Textgestaltung im untenstehenden Abschnitt 'Orthographisches' (S. X-XVI) zusammengefaßt; den apparatus criticus hat Almut Fries neu gestaltet (s. S. XVII); der Testimonien-Apparat konnte hier entfallen. Einen Abriß der Überlieferungsgeschichte bietet das Kapitel 'Zur Geschichte des Textes' (GT) im Prolegomena-Band. - Eine typographische Besonderheit der vorliegenden Ausgabe stellt die Hervorhebung der direkten Reden durch Kursivsatz dar. Damit wird dem 'Fokalisations'-Unterschied zwischen Erzähler-Text und Figuren-Sprache Rechnung getragen, dem im Kommentar besondere Aufmerksamkeit geschenkt wird (s. dazu das Prolegomena-Kapitel 'Homerische Poetik in Stichwörtern' s.v. 'Sekundäre Fokalisation').

\section{Abkürzungen und Siglen}

GRAMMATICI

$\begin{array}{ll}\text { Ammon } & \text { Ammonius } \\ \text { ApS } & \text { Apollonius Sophista } \\ \text { Ar } & \text { Aristarchus } \\ \text { Arab }^{\text {b }} & \text { Didymi exemplaria duo Aristarchea } \\ \text { Arn } & \text { Aristonicus } \\ \text { Arph } & \text { Aristophanes Byzantius } \\ \text { Did } & \text { Didymus } \\ \text { DSid } & \text { Dionysius Sidonius } \\ \text { Epm } & \text { Epimerismi Homerici } \\ \text { Hdn } & \text { Herodianus } \\ \text { Nic } & \text { Nicanor } \\ \text { Ptol } & \text { Ptolemaeus Ascalonita } \\ \text { Tyr } & \text { Tyrannio } \\ \text { Zen } & \text { Zenodotus }\end{array}$


FONTES ANTIQUIORES

$\begin{array}{ll}\mathfrak{P} & \dot{\eta} \pi \mathrm{o} \lambda \hat{\sigma} \sigma \tau \chi \mathrm{x} \sigma \varsigma \\ \mathrm{pp} & \text { papyri } \\ \mathrm{p} & \text { papyrus una } \\ \mathrm{sch} & \text { scholia } \\ \mathrm{sch}^{\mathrm{bT}} & \text { scholia in libris B C E F T tradita } \\ \mathrm{sch}^{\mathrm{D}} & \text { scholia minora quae dicuntur } \\ \mathrm{sch}^{\mathrm{G}} & \text { scholia in libro G tradita } \\ \mathrm{tt} & \text { testimonia auctorum antiquorum } \\ \mathrm{t} & \text { testimonium auctoris unius }\end{array}$

\section{CODICES AEVI MEDII}

$\begin{array}{ll}\text { A } & \text { Marc. gr. 822 (olim 454), saec. x } \\ \text { B } & \text { Marc. gr. 821 (olim 453), saec. xi } \\ \text { C } & \text { Laur. 32.3, saec. xi-xii } \\ \text { D } & \text { Laur. 32.15, saec. x (sed in Il. 1-4 saec. xii) } \\ \text { E } & \text { Scorial. Y.I.1 (291), saec. xi (sed in Il. 1.29-200 saec. xii) } \\ \text { F } & \text { Scorial. } \Omega . I .12 \text { (509), saec. xi } \\ \text { G } & \text { Genav. 44, saec. xiii } \\ \text { H } & \text { Vindob. phil. gr. 117, saec. xiii } \\ \text { O } & \text { Oxon. Bodl. New College 298, saec. xiii } \\ \text { R } & \text { Oxon. Bodl. Auct. T.2.7, saec. xii } \\ \text { T } & \text { Lond. Bibl. Brit. Burney 86, ann. 1059 } \\ \text { V } & \text { Vat. gr. 26, saec. xiii } \\ \text { X } & \text { Sinaiticus, saec. ix (fragmentum: } \Delta \text { 367-?) } \\ \text { Y } & \text { Paris. suppl. gr. 663, saec. xi (fragmenta, excerpta) } \\ \text { W } & \text { Vat. gr. 1319, saec. xii } \\ \mathrm{Z} & \text { Rom. Bibl. Nat. gr. 6 + Matrit. 4626, saec. ix (lemmata et sch }{ }^{\text {D) }} \text { ) } \\ b & \text { archetypus librorum B C E } \\ h & \text { archetypus librorum M N P } \\ \Omega & \text { libri A D B C E F T (Y) R W G } \\ \Omega * & \text { tot horum quot non singuli laudantur } \\ \text { r, rr } & \text { liber recentior unus vel plures }\end{array}$

\section{SigLA CETERA}

$\begin{array}{ll}\mathrm{A}^{\mathrm{a}} & \text { A ante correcturam } \\ \mathrm{A}^{\mathrm{a}} ? & \text { fortasse A ante correcturam } \\ \mathrm{A}^{\mathrm{c}} & \text { A post correcturam } \\ \mathrm{A}^{\gamma \rho} & \text { varia lectio in A adscripta } \\ \mathrm{A}^{\lambda} & \text { lemma scholii in A }\end{array}$




\begin{tabular}{|c|c|}
\hline $\mathrm{A}^{\mathrm{m}}$ & A in margine \\
\hline $\mathrm{A}^{\mathrm{s}}$ & A super lineam \\
\hline $\mathrm{A}^{\mathrm{uv}}$ & A ut videtur \\
\hline $\mathrm{A}^{\mathrm{x}}$ & A ante vel post correcturam (incertum utrum) \\
\hline [ ] & textus papyri periit \\
\hline$\llbracket \rrbracket$ & delevit scriba \\
\hline\{\} & interpolata videntur \\
\hline$\dagger \dagger$ & corrupta videntur \\
\hline add. & addidit, addiderunt \\
\hline ap. & apud \\
\hline ath. & $\grave{\eta} \theta \dot{\varepsilon} \tau \eta \kappa \varepsilon$ \\
\hline damn. & damnavit \\
\hline def. & defendit \\
\hline del. & delevit \\
\hline deprec. & deprecatur \\
\hline fort. & fortasse \\
\hline hab. & habet, habent, habuit, habuerunt \\
\hline mg. & in margine \\
\hline m. rec. & manus recentior \\
\hline nol. & noluit \\
\hline nov. & novit \\
\hline om. & omisit, omiserunt \\
\hline prob. & probavit \\
\hline rest. & restituit \\
\hline susp. & suspicatus est (falsum esse) \\
\hline v.l. & varia lectio \\
\hline
\end{tabular}




\section{Orthographisches (ORTH)}

\section{ASPIRATION}

$\dot{\varepsilon} \sigma \pi$ ó $\mu \eta v$ (statt $\dot{\varepsilon} \sigma \pi$-): $\dot{\varepsilon}$ - ist das Augment, $\sigma \pi$ - Schwundstufe zum * $\sigma \varepsilon \pi->\dot{\varepsilon} \pi$ - des

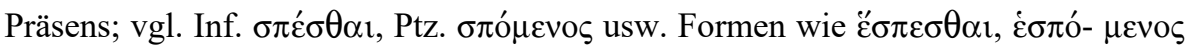
sind wohl erst nachhomerisch.

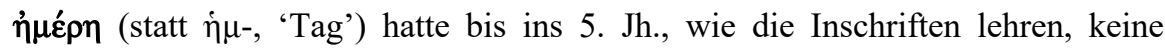
Aspiration. Diese stammt vielleicht von dem Adjektiv $\dddot{\eta} \mu \varepsilon \rho \varsigma^{\prime}$ 'zahm' her (THREATTE 1980, 500).

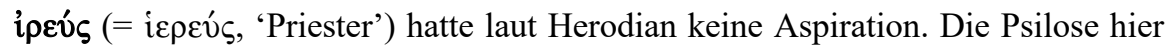
sowie bei 'p Ionisch gemeinsame Dialekt-Erscheinung (in diesen Gegenden sagte man ipó $\varsigma$ statt iєрós).

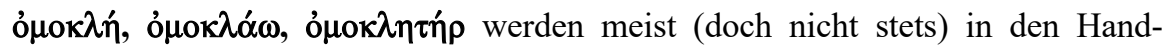
schriften aspiriert, offenbar in dem Glauben, daß das Element ópo- darin steckt. Man hat es dagegen ansprechend einem indo-iranischen Wort áma- 'Kraft' gleichgestellt (DELG): falls mit Recht, hatte es von Haus aus kein $h$.

\section{AKZENTUATION}

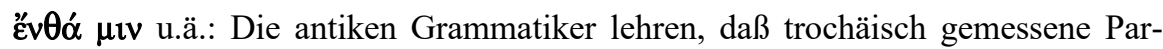
oxytona vor Enklitika einen zweiten Akut tragen (Herodian 1.563.2f.), und diese Praxis wird in den älteren Handschriften (z.B. 4.247, 4.539, 5.305) weitgehend eingehalten. Sie lehren weiter, daß alle Paroxytona den zweiten Akut erhalten, wenn das

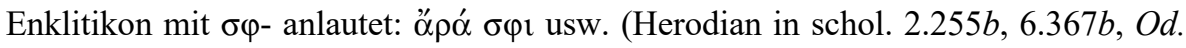
12.40; WACKeRNAGEL [1893] 1953, 1095f.; WeSt 1966, 440-442; vgl. SCHW. 1.391). Auch dies ist in einigen mittelalterlichen Handschriften noch erhalten $(9.99,11.807$, 14.384).

Die zusammengesetzten Adverbien und Präpositionen $\dot{\alpha} \pi \circ \pi \rho \circ \delta 1 \varepsilon \xi \pi \alpha \rho \varepsilon \xi \dot{v} \pi \varepsilon \xi$ $\delta ı \alpha \pi \rho 0 \pi \varepsilon \rho \iota \pi \rho$ wurden von den antiken Grammatikern verschieden beurteilt; Aristarch hat anscheinend die beiden Bestandteile jeweils separat geschrieben und

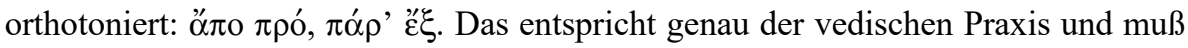
alt sein. Diese Zusammensetzungen wurden aber offenbar schon lange vor Homer als

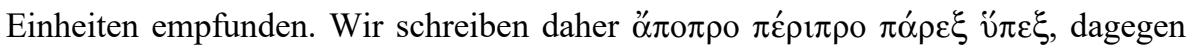

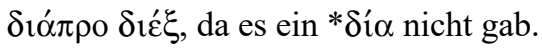

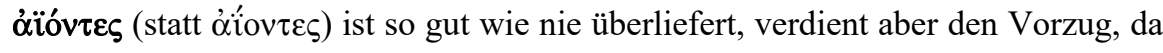
SCHULZE (1888) 1934, 345, die Form überzeugend für einen Aorist erklärt hat. 


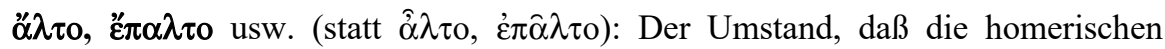

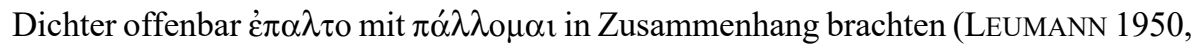
60-62), so als wäre es als "̌- $\pi \alpha \lambda \tau$ o aufzufassen, setzt voraus, daß sie das $\varepsilon$ betont und das $\alpha$ kurz hörten: also augmentlos $\varepsilon^{\prime} \pi-\breve{\alpha} \lambda \tau$, nicht (äolisch) augmentiert $\dot{\varepsilon} \pi-\hat{\alpha} \lambda \tau$ o. Für das Simplex ö $\lambda \tau$ o ist das gleiche anzunehmen.

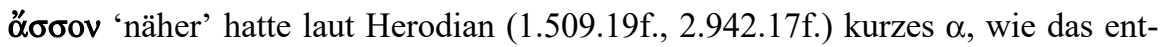

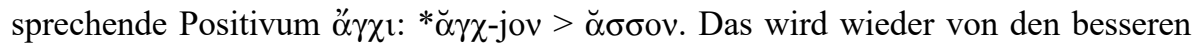
Handschriften geboten (ALLEN 1931, 242); die gewöhnliche Form $\hat{\alpha} \sigma \sigma o v$ scheint attisch (WACKERNAGEL [1914] 1953, 1181f.; 1916, 13; CHANTR. 1.190, 256; anders LfgrE s.v. ő $\gamma \chi 1$; SIHLER 1995, 363). Ist das richtig, so ist Friedrich Blass (KÜHNERBLASS 1.555) recht zu geben, der statt der fast einheitlich überlieferten Formen

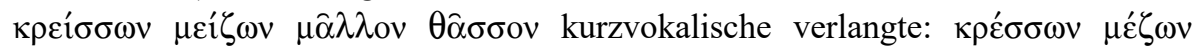

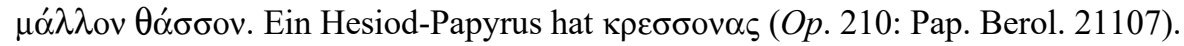

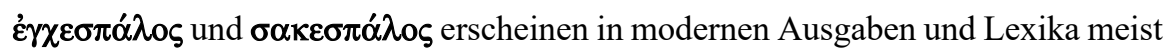
als Proparoxytona. Wegen der aktivischen Bedeutung ('den Speer bzw. den Schild schwingend') ist jedoch Paroxytonese zu erwarten, und tatsächlich wird diese nicht nur von fast allen guten Handschriften geboten $(2.131,14.449,15.605)$, sondern im

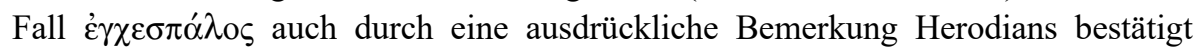

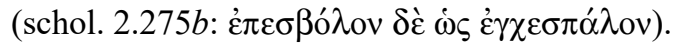

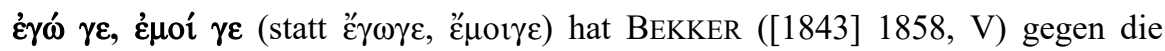
Überlieferung wiederhergestellt; die zweitgenannten Formen sind Ergebnis einer dem Attischen eigentümlichen Regel (s. Ap. Dysc., Pron. 49.9ff., Adv. 181.30, Synt. 138.9), wonach Wörter der Messung $\smile \_\smile$, die auf der zweiten Silbe betont waren,

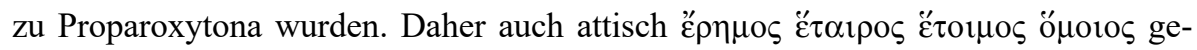

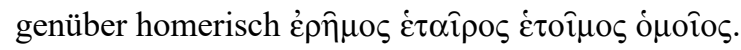

Die beiden Verben $\varepsilon$ i $\mu \imath$ 'sum' und $\varphi \eta \mu \imath$ erscheinen im Präsens meist als Enklitika:

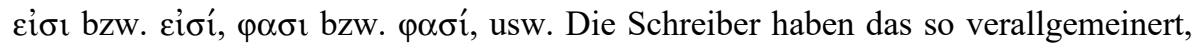
daß sie auch am Satzanfang cioí, $\varphi \alpha \sigma i ́$ betonten. Das ist verfehlt, denn in dieser Stellung mußten orthotone Formen stehen, d.h. nicht nur "̌ $\sigma \tau$ ( (das sich weiterhin

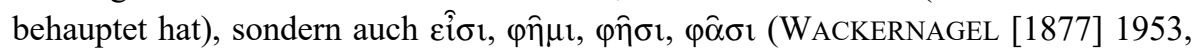
1068; VENDRYÈS 1945, 108. 110). - $ү \hat{\eta} \mu$ 1 2.350 wird übrigens vom Grammatiker Tyrannion befürwortet.

Wenn das Adverb $\varepsilon \hat{\text { z }}$ zweisilbig gemessen wird, schreiben die Herausgeber meist

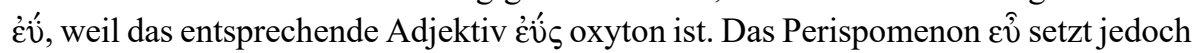
¿̌ü voraus, wie Apollonios Dyskolos ( $A d v$. 200.20f., 29ff.) und Herodian (1.506.21ff.) akzentuierten und der Venetus A $(1.253,2.78)$ bietet: im Adverb wird der Akzent

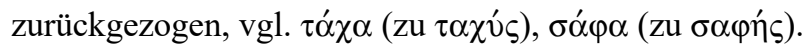

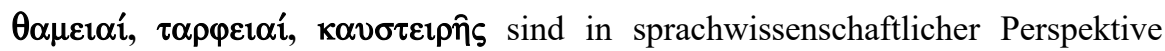
Altertümlichkeiten, die von Aristarch bezeugt werden und sich offenbar durch 
Rhapsodenüberlieferung, trotz des Ausgleichszwangs der Analogie, bis in die hellenistische Zeit erhalten hatten (WACKERNAGEL [1893] 1953, 1103; [1914] 1953, 1176 bzw. 1126 u. 1175f.; SCHW. 1.385, 474).

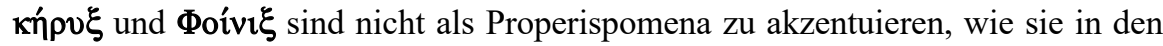
meisten Handschriften erscheinen und wie selbst Herodian es für richtig hielt, da der zweite Vokal in beiden Wörtern lang ist, wie die Deklination zeigt (ки́рขко usw.); daß er sich im Nominativ verkürzen sollte, ist undenkbar (Näheres s. WEST 1990, XLVIII).

$\pi \mathbf{i} \pi \tau \varepsilon$ wird richtig sein, nicht $\pi i ̂ \tau \tau \varepsilon$, wie meist gedruckt. $\pi \mathrm{l}$ - ist Reduplikationssilbe, also kurz, $-\pi \tau$ - ist Schwundstufe zu $\pi \varepsilon \tau-$; vgl. $\mu i^{\prime}-\mu v \omega(\mu \varepsilon v-), \tau i^{\prime}-\kappa \tau \omega(<* \tau i-\tau \kappa \omega: \tau \varepsilon \kappa-$ ), $\gamma^{\prime}-\gamma v 0 \mu \alpha \imath(\gamma \varepsilon v-)$. Man hat $\pi \hat{\imath} \pi \tau \varepsilon$ geschrieben wegen $\hat{\rho} i \pi \tau \varepsilon$, das wurzellanges $\imath$ hat.

$\tau \omega ́$ 'darum' wird meist $\tau \hat{\omega}$ oder $\tau \hat{\emptyset}$ geschrieben. Das Iota jedenfalls ist sekundär, da es sich um einen alten Instrumentalis oder Ablativus handelt. Die antiken Ansichten über den Akzent sind schwer auszumachen; Apollonios Dyskolos scheint den Akut befürwortet zu haben.

In der Wendung $\hat{\omega} \pi$ ó $\pi$ or bieten die meisten Handschriften und Herausgeber die Akzentuierung $\hat{\omega}$, wie sie für einen Ausruf geeignet wäre. Herodian hat das Wort aber gleich wie in $\hat{\omega} \varphi$ pí $\mathrm{dor}$ akzentuiert. Sich darüber hinwegzusetzen besteht kein Grund.

\section{KONTRAHIERTES EO}

Die Kontraktion der Vokale eo bzw. $\varepsilon \bar{o}$ wird in der homerischen Überlieferung häufig durch die Schreibung $\varepsilon v$ wiedergegeben: $\dot{\varepsilon} \mu \varepsilon \hat{v}, \sigma \varepsilon v, \kappa \alpha \lambda \varepsilon \hat{v} v \tau$, usw. Das kann aber schwerlich für den Urtext angenommen werden, da die ionischen Inschriften lehren, daß diese Schreibweise erst um 400 v. Chr. üblich wurde. Davor hat man weiterhin eo geschrieben, nicht aus philologischen Gründen, sondern offenbar deswegen, weil man dieses diphthongierte عo vom ererbten Diphthong $\varepsilon v$ in der Aussprache noch unterschied (Beispiele für in Homer-Handschriften erhaltenes -

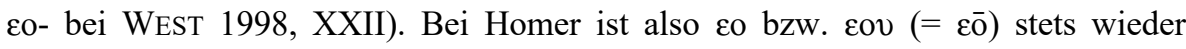

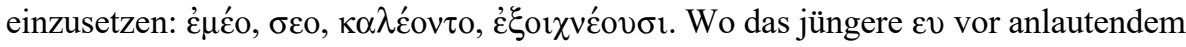
Vokal gekürzt erscheint (scheinbar correptio epica), wird man elidiertes -عo annehmen, z.B. 10.146 ह̌ $\pi \varepsilon '$ (so Aristarch) statt $\check{\pi} \pi \varepsilon \breve{v}$ (Handschriften), $14.95 \sigma \varepsilon$ ' (Zenodot) statt $\sigma \varepsilon \breve{v}$. 


\section{VERSCHIEDENES}

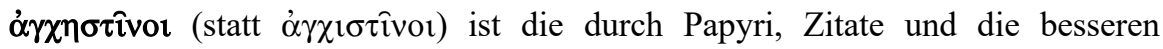
Handschriften empfohlene Schreibung (5.141, 17.361). Die Bildung ist dunkel; im-

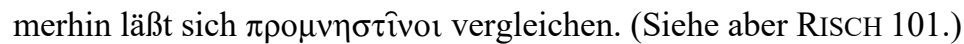

$\zeta \omega ́ v v \mu \imath$ (statt $\zeta \dot{v} v v v \mu \imath$ ) ist sprachgeschichtlich die ältere Form und wohl die homerische, wenngleich das nicht strikt beweisbar ist (vgl. WACKERNAGEL 1916, 17; CHANTR. 1.175; CASSIO 1991/93, 201f.). Einfaches - $v$ - ist in einigen Handschriften vorhanden (codd. DT in 5.857, DTG in 10.78, DCETG in 11.15), was freilich nicht viel besagt.

$-\eta \sigma \mathrm{\imath}$ (statt $-\eta \sigma \mathrm{l})$ in der 3. Sg. Konj. ist die richtige Schreibung, vgl. das bekannte Epigramm auf dem 'Nestor-Becher' aus Pithekoussai (CEG 454, um 725 v. Chr.): hos $\delta^{\prime} \alpha \nu \tau \bar{o} \delta \varepsilon \pi \bar{\varepsilon} \sigma 1 \pi \circ \tau \bar{\varepsilon} \rho 1 \bar{\kappa} \kappa \tau \lambda$.

Oví (statt $\theta \dot{\omega} \omega$ ) in der Bedeutung 'rasen, toben' ist vielfach durch Papyri und andere gute Quellen bezeugt und auch sprachwissenschaftlich berechtigt (SCHULzE 1892,314-316).

Wo Verben auf - $i \zeta \omega$ ihr Futur auf $-\imath \omega$ bilden, ist das wohl nicht, wie in der späteren Sprache, als eine kontrahierte Form aufzufassen $(-1 \hat{\omega})$, sondern aus *-í⿴囗十 herzuleiten und -í $\omega$ zu schreiben (WACKERNAGEL [1893] 1953, 828-830; CHANTR. 1.451). Dementsprechend hat Wackernagel in $10.331 \dot{\alpha} \gamma \lambda \alpha \dot{i} \varepsilon \sigma \theta \alpha$ für das überlieferte $\dot{\alpha} \gamma \lambda \alpha \ddot{\imath} \varepsilon i \tilde{\sigma} \theta \alpha$ eingesetzt (a.O. 829).

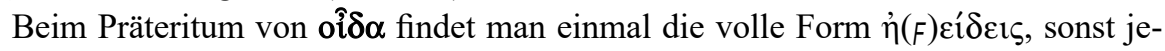

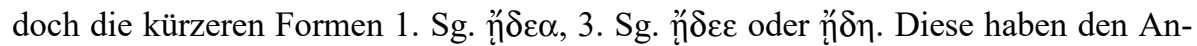
schein, aus regelrechter Kontraktion entstanden zu sein $(\dot{\eta} \varepsilon \imath>\grave{\eta})$. Vor ihnen (sofern sie nicht am Versanfang stehen) liegt jedoch immer Hiat vor, als lauteten sie mit Digamma an. Das trifft jedoch nur für die nicht augmentierte Form $(F) \varepsilon i \delta$ - zu. Diese also ist offenbar die dem Dichter geläufige; demnach ist stets $\varepsilon \varepsilon^{\prime} \delta \varepsilon \alpha$, $\varepsilon \varepsilon^{\prime} \delta \varepsilon \varepsilon$, $\varepsilon^{\prime} \delta \emptyset \eta$ schreiben (WACKERNAGEL [1878] 1979, 1519). Im Konjunktiv hat Tyrannion wohl

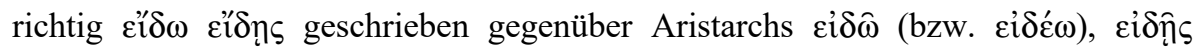
(WACKERNAGEL 1916, 73).

ó $\pi \dot{i} \sigma \sigma \omega \tau \rho \alpha$ war an mehreren Stellen eine antike Variante für das geläufige

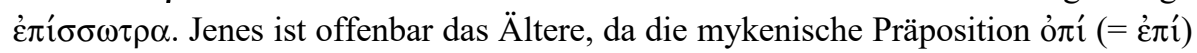
darin noch bewahrt ist.

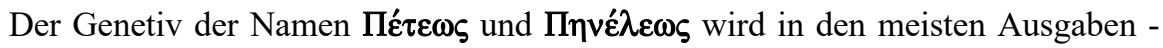

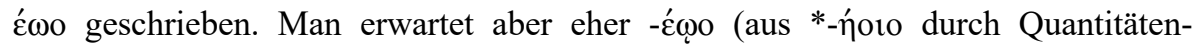
metathese), und dafür spricht die Autorität Aristarchs sowie das Zeugnis mehrerer Papyri.

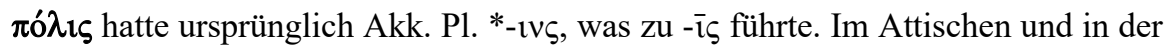
Koine ist $-\bar{i} \varsigma$ durch das vom Nominativ übernommene - $\varepsilon 1 \zeta$ verdrängt worden. Dies herrscht weitgehend auch in der Homer-Überlieferung vor. Doch hier und da gibt es Spuren von $\pi$ ó $\lambda i ̄$ (codd. AV in 2.648, gegen die Papyri, u.a.; s. WeSt 1998, XXXIV), 
und man wird kaum fehlgehen, wenn man diese Form auch sonst einsetzt. Analog

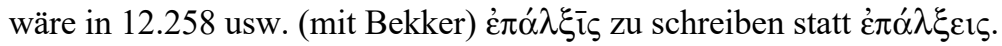

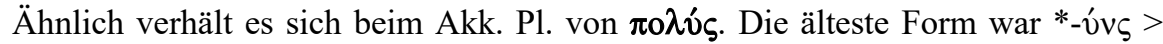
-v́s. Später ist $\pi \mathrm{o} \lambda \hat{\varepsilon}^{\alpha} \alpha \varsigma$ aufgekommen und noch später $\pi \mathrm{o} \lambda \varepsilon \hat{\imath} \varsigma$. Diese Formen sind in der Homer-Überlieferung normal, auch wo zweisilbige Messung geboten ist. Doch an

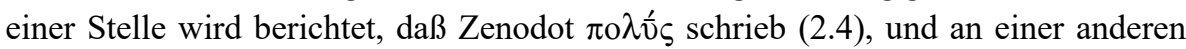
(1.559) scheint ein Papyrus diese Lesart zu bieten. Das kann nur auf alter, echter Überlieferung beruhen. Die altertümliche Form wird also auch sonst wiederherzustellen sein.

$\sigma \mu \omega ́ \delta \imath \xi$ (statt $-1 \xi$ ), wie bei Hesych und Doxapatres zu 2.267, muß der richtige Nominativ des Worts sein, da der Stamm $\sigma \mu \omega ́ \delta \imath \gamma \gamma-$ ist (23.716 $\sigma \mu \omega \dot{\delta} \imath \gamma \gamma \varepsilon \varsigma)$. Der Akut ist trotz des kurzen Iota berechtigt, da das $\imath \gamma$ [iñ], Vokal + Sonant, einem Diphthong gleichkommt (VENDRYÈs 1945, 50).

Die Partikel $\tau \alpha \rho$, die mehrmals hinter einem Fragewort steht ( $\tau$ í $\varsigma \alpha \rho, \pi \omega \hat{\varsigma} \tau \alpha \rho \rho$ usw.), ist nicht in $\tau$ ' ö $\rho$ zu zerlegen, denn erstens wäre $\tau \varepsilon$ in einer Frage nicht am Platz, und zweitens heißt es (nach Fragewort) nie $\tau \alpha$ ó $\rho \alpha$ bzw. $\tau$ ' ’’ $\rho \alpha$, obwohl diese Verbindung in der Erzählerrede üblich ist (1.465 = 2.428, 11.254, 15.397, 18.37 usw.) und zumindest in 1.123 und 2.761 auch metrisch gepaßt hätte. Schon Apollonios Dyskolos und Herodian haben $\tau \alpha \rho$ für éin Wort erklärt. Es ist spezifisch homerisch, offenbar ein ganz altes Überbleibsel. WATKINS 1995, 150, hat es treffend mit der luwischen Partikel tar gleichgesetzt, die in der Verbindung kwis tar 'wer immer' bezeugt ist.

$\tau \varepsilon ́ \sigma \sigma \varepsilon \rho \varepsilon \varsigma$ (statt $\tau \varepsilon ́ \sigma \sigma \alpha \rho \varepsilon \varsigma$ ) ist eine ionische Dialektform (THUMB-SCHERER 281f.), die häufig von den Homer-Papyri, mitunter auch vom Venetus A geboten wird und somit sehr gute Chancen hat, echt zu sein.

$\tau \rho \hat{i} \varsigma$ bzw. $\tau \rho \bar{i} \varsigma$ (aus * $\tau \rho^{\prime} v_{\zeta}$ ) war nach allgemeiner Überzeugung die alte AkkusativForm von $\tau \rho \varepsilon i \varsigma$ (CHANTR. 1.260). Die Homer-Überlieferung bietet fast ausnahmslos $\tau \rho \varepsilon \hat{i} \varsigma$; nur an einer Stelle (2.671) scheint ein Papyrus $\tau \rho[1] \varsigma$ gehabt zu haben, was freilich ein trivialer Fehler sein kann. Trotzdem bleibt der Verdacht, daß Akk. $\tau \rho \varepsilon \hat{\varsigma} \varsigma$ eine Modernisierung darstellt.

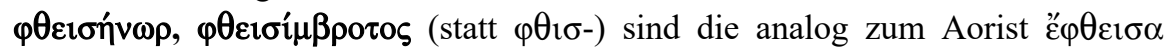
(WACKernagel 1916, 75f.) zu erwartenden Bildungen. So haben Tryphon und Didymos geschrieben und als überliefert vorgefunden (s. Philox. Gramm. fr. 619 Theodoridis), und die Schreibung taucht gelegentlich auch in Papyri auf.

$\chi \varepsilon i ́ \lambda$ เor (statt $\chi$ í $\lambda$ 1or) war die gemeingriechische Form des Zahlworts, $\chi$ í $\lambda$ ıor war spezifisch attisch. Auch in diesem Fall sind Spuren der echten (vorattischen) Überlieferung in den Papyri erhalten geblieben (s. zu 7.471, 11.244). Hinzu kommt, daß Aristarch die Komposita $\varepsilon \dot{v} v \varepsilon \alpha ́ \chi(\varepsilon) 1 \lambda o 1$ und $\delta \varepsilon \kappa \alpha ́ \chi(\varepsilon) 1 \lambda o 1(5.860,14.148)$ als 'neun bzw. zehn $\chi \varepsilon i ́ \lambda \eta$ habend' erklärte; er hat also offenbar - $\chi \varepsilon \imath \lambda$ or gelesen. 


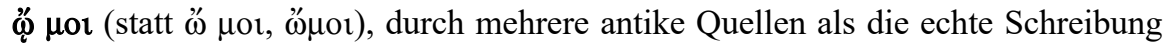
bezeugt, steht noch in den besten mittelalterlichen Handschriften von Homer, Aischylos und Sophokles (s. dazu WEST 1990, LIII).

\section{Zu weiteren Einzelheiten s. WEST 1998.}

\section{BIBLIOGRAPHISCHE ABKÜRZUNGEN}

Allen 1931

Bekker (1843) 1858

Cassio 1991/93

$C E G$

Chantr.

DELG

Kühner-Blass

Leumann 1950

LfgrE

Risch

Schulze (1888) 1934

Schulze 1892

Schw.

Sihler 1995

Threatte 1980

Thumb-Scherer

Vendryès 1945
Allen, T.W.: Homeri Ilias, I: Prolegomena, Oxford 1931.

Bekker, I.: Carmina Homerica. Emendabat et annotabat I. B., I: Ilias, Bonn ${ }^{2} 1858\left({ }^{1} 1843\right)$.

Cassio, A.C.: La più antica iscrizione greca di Cuma e $\tau^{\prime} v(v) v \mu \alpha \imath$ in Omero, in: Die Sprache 35, 1991/93, 187-207.

Hansen, P.A.: Carmina epigraphica Graeca (Texte und Kommentare, 12 u. 15), Berlin/New York 1983-1990 (2 Bde.).

Chantraine, P.: Grammaire homérique, Paris ${ }^{6} 1986-88$ ( $\left.{ }^{1} 1942-53\right)(2$ Bde.).

Chantraine, P.: Dictionnaire étymologique de la langue grecque. Histoire des mots, Paris 1968-1980.

Kühner, R. / Blass, F.: Ausführliche Grammatik der griechischen Sprache. 1. Teil: Elementar- und Formenlehre, Hannover ${ }^{3} 1890-1992$ (2 Bde.; $\left.{ }^{1} 1834-35\right)$.

Leumann, M.: Homerische Wörter (Schweizerische Beitr. zur Altertumswiss., 3), Basel 1950 (Nachdruck Darmstadt 1993).

Lexikon des frühgriechischen Epos. Begründet von Bruno Snell. Im Auftrag der Akademie der Wissenschaften in Göttingen vorbereitet und hrsg. vom Thesaurus Linguae Graecae, Göttingen 1955ff.

Risch, E.: Wortbildung der homerischen Sprache, Berlin/New York ${ }^{2} 1974\left({ }^{1} 1937\right)$.

Schulze, W.: Zwei verkannte Aoriste, in: ders., Kleine Schriften, hrsg. vom Indogermanischen Seminar der Universität Berlin, Göttingen o. J. (1934) 330-349 (urspr. in: KZ 29, 1888, 230-255).

Schulze, W.: Quaestiones Epicae, Gütersloh 1892.

Schwyzer, E. / Debrunner, A. / Georgacas, D.J. / Radt, F. und S.: Griechische Grammatik (Handbuch der Altertumswissenschaft, 2.1.14), München 1939-1994 (4 Bde.).

Sihler, A.L.: New Comparative Grammar of Greek and Latin, New York 1995.

Threatte, L.: The Grammar of Attic Inscriptions, I: Phonology, Berlin/ New York 1980.

Thumb, A. / Scherer, A.: Handbuch der griechischen Dialekte, 2. Teil, Heidelberg ${ }^{2} 1959\left({ }^{1} 1909\right)$.

Vendryès, J.: Traité d'accentuation grecque, Paris 1945. 
Wackernagel (1877) 1953 Wackernagel, J.: Der griechische Verbalakzent, in: Wackernagel 1953 (Bd. 2), 1058-1071 (urspr. in: KZ 23, 1877, 457-470).

Wackernagel (1878) 1979 Wackernagel, J.: Die epische Zerdehnung, in: Wackernagel 1979, 1512-1565 (urspr. in: Beiträge zur Kunde der indogermanischen Sprachen 4, 1878, 259-312).

Wackernagel (1893) 1953 Wackernagel, J.: Beiträge zur Lehre vom griechischen Akzent, in: Wackernagel 1953 (Bd. 2), 1072-1107 (urspr. in: Programm zur Rektoratsfeier der Universität Basel, Basel 1893, 3-38).

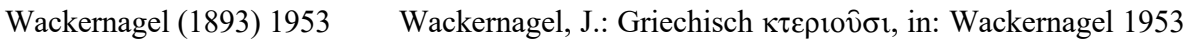
(Bd. 1), 828-830 (urspr. in: IF 2, 1893, 141-154).

Wackernagel (1914) 1953 Wackernagel, J.: Akzentstudien II; Akzentstudien III, in: Wackernagel 1953 (Bd. 2), 1122-1153; 1154-1187 (urspr. in: GN 1914, 20-51; 97-130).

Wackernagel 1916 Wackernagel, J.: Sprachliche Untersuchungen zu Homer (Forschungen zur griechischen und lateinischen Grammatik, 4), Göttingen 1916 (Nachdruck 1970).

Wackernagel 1953 Wackernagel, J.: Kleine Schriften, Bd. 1-2, hrsg. von der Akad. der Wiss. zu Göttingen, Göttingen 1953 u.ö.

Wackernagel 1979 Wackernagel, J.: Kleine Schriften, Bd. 3, hrsg. von B. Forssman im Auftrage der Akad. der Wiss. zu Göttingen, Göttingen 1979.

Watkins 1995 Watkins, C.: How to Kill a Dragon. Aspects of Indo-European Poetics, New York 1995.

West 1966 West, M.L.: Hesiod, Theogony. Edited with Prolegomena and Commentary, Oxford 1966 u.ö.

West 1990 West, M.L.: Praefatio, in: Aeschyli tragoediae cum incerti poetae Prometheo. Edidit M.L. W., Stuttgart 1990, III-LV.

West 1998 West, M.L.: Praefatio, in: Homeri Ilias. Recensuit / testimonia congessit M.L. W., Bd. 1, Stuttgart/Leipzig 1998, V-XXXVII. 